\title{
Simulation of hydrodynamically interacting particles near a no-slip boundary
}

\author{
James W. Swan and John F. Brady \\ Division of Chemistry and Chemical Engineering, California Institute of Technology, \\ Pasadena, California 91125, USA
}

(Received 9 May 2007; accepted 6 September 2007; published online 14 November 2007)

\begin{abstract}
The dynamics of spherical particles near a single plane wall are computed using an extension of the Stokesian dynamics method that includes long-range many-body and pairwise lubrication interactions between the spheres and the wall in Stokes flow. Extra care is taken to ensure that the mobility and resistance tensors are symmetric, positive, and definite-something which is ineluctable for particles in low-Reynolds-number flows. We discuss why two previous simulation methods for particles near a plane wall, one using multipole expansions and the other using the Rotne-Prager tensor, fail to produce symmetric resistance and mobility tensors. Additionally, we offer some insight on how the Stokesian dynamics paradigm might be extended to study the dynamics of particles in any confining geometry. (C) 2007 American Institute of Physics.
\end{abstract}

[DOI: $10.1063 / 1.2803837$ ]

\section{INTRODUCTION}

Computational simulations of colloidal particles typically study the properties of unbounded suspensions. However, suspensions are often bounded by walls, and a rich assortment of phenomena including templated self-assembly ${ }^{1}$ and shear-induced resuspension ${ }^{2}$ arise precisely because hydrodynamic flows are intimately coupled to the interactions of particles with boundaries. For colloidal particles in lowReynolds-number flows, a mobility tensor linearly couples the forces and torques on particles to their velocities and provides a complete characterization of the hydrodynamic interactions among particles. In principle, this tensor includes the effects of a plane wall on the hydrodynamics, but including these effects is not trivial.

It is often the case that details of the fluid motion throughout a suspension are ignored in simulations of many hydrodynamically interacting particles. In fact, the choice to embed the fluid flow implicitly in mobility and resistance tensors allows for the rapid simulation of the dynamics of particles numbering from just a few up into the thousands. Even though the detailed fluid velocity field is discarded in favor of these tensors, the Stokes equations dictate specific properties that must emerge from the hydrodynamic interactions among particles. Principally, the mobility and resistance tensors must be symmetric and positive-definite. While models can be constructed which have neither of these properties, the physics governing the Brownian motion of hydrodynamically interacting particles require that the mobility and resistance tensors have orthogonal eigenvectors and strictly positive eigenvalues. ${ }^{3}$

We have a twofold motivation for developing a new approach to this problem. First, in order to study the Brownian motion of colloidal particles near a wall, it is essential that the mobility tensor be symmetric. ${ }^{3}$ Second, the relatively simple and physically intuitive strategy first used in unbounded Stokesian dynamics simulations, ${ }^{4}$ in which the mo- bility tensor is constructed directly from Faxén formulas, is easy to leverage and extend to study a number interesting problems. Although others have developed simulations of colloidal particles near a single wall, these studies lack either the crucial symmetry of the method herein ${ }^{5,6}$ or the physical and mathematical straightforwardness of the original Stokesian dynamics technique. ${ }^{7,8}$ In Sec. II we discuss our approach to computing the mobility tensor from multipole expansions and Faxén formulas. Included is a discussion of why two previous approaches failed to compute symmetric mobility tensors and cannot be used for Brownian dynamics simulations. Examples are given illustrating the importance of the symmetry of the resistance and mobility tensors.

\section{THEORY AND METHOD}

Consider the problem of particles moving in an ambient flow field $\mathbf{u}^{\infty}(\mathbf{x})$. The grand mobility tensor, denoted by $\mathcal{M}$, couples the velocity moments of the particles relative to this imposed flow $\left(\mathbf{U}-\mathbf{U}^{\infty}, \boldsymbol{\Omega}-\boldsymbol{\Omega}^{\infty},-\mathbf{E}^{\infty}, \ldots\right)$ to the moments of the hydrodynamic force density on the particle surfaces $(\mathbf{F}, \mathbf{T}, \mathbf{S}, \ldots)$,

$$
\left(\begin{array}{c}
\mathbf{U}-\mathbf{U}^{\infty} \\
\mathbf{\Omega}-\mathbf{\Omega}^{\infty} \\
-\mathbf{E}^{\infty} \\
\vdots
\end{array}\right)=-\left(\begin{array}{cccc}
\mathbf{M}_{U F} & \mathbf{M}_{U L} & \mathbf{M}_{U S} & \cdots \\
\mathbf{M}_{\Omega F} & \mathbf{M}_{\Omega L} & \mathbf{M}_{\Omega S} & \cdots \\
\mathbf{M}_{E F} & \mathbf{M}_{E L} & \mathbf{M}_{E S} & \cdots \\
\vdots & \vdots & \vdots & \ddots
\end{array}\right) \cdot\left(\begin{array}{c}
\mathbf{F} \\
\mathbf{L} \\
\mathbf{S} \\
\vdots
\end{array}\right)
$$

where $\mathbf{U}$ and $\boldsymbol{\Omega}$ are the translational and rotational velocities of the particles, $\mathbf{U}^{\infty}, \mathbf{\Omega}^{\infty}$ and $\mathbf{E}^{\infty}$ are velocity, vorticity, and rate of strain of the imposed flow at the particles' centers and $\mathbf{F}, \mathbf{L}$, and $\mathbf{S}$ are the total hydrodynamic force, torque, and stresslet on the particles. It follows directly from the Stokes equations and the reciprocal theorem that the grand mobility tensor is always symmetric, positive, and definite. ${ }^{9}$ 


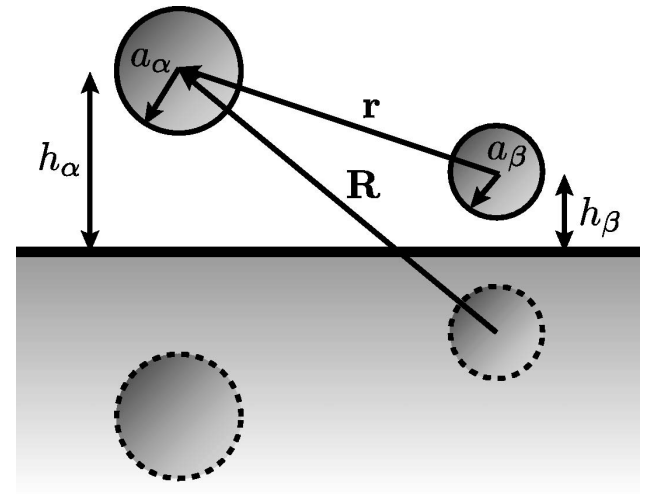

FIG. 1. The interactions between a pair of spheres $(\alpha$ and $\beta)$ near a plane wall where $h_{\alpha}=x_{3}^{(\alpha)}-H$ and $h_{\beta}=x_{3}^{(\beta)}-H$.

The structure of Eq. (1) and its symmetries applies equally well to particles in an unbounded fluid as to particles adjacent to a wall or walls. Shown in Fig. 1 is a sample configuration of a pair of spheres $(\alpha, \beta)$ above a plane wall. The hydrodynamic flows generated by motion of these particles near the wall must produce symmetric interactions between the pair as well as between each particle and the wall. Two natural although not necessarily intuitive consequences are that for a single particle near a wall, there exists couplings between torque and translation and between force and rotation, and that these couplings are symmetric $\left(\mathbf{M}_{U L}=\mathbf{M}_{\Omega F}^{T}\right)$. Some bacteria actually put these properties to work when swimming near a plane wall, smoothing out their almost chaotic run and tumble paths through the fluid. ${ }^{10}$ Any model of the motion of particles in the low-Reynoldsnumber limit, no matter how approximate, should at a minimum preserve these traits as they are fundamental to the physics of Stokes flow. For instance, one of the reasons why the full Rotne-Prager approximation for $\mathbf{M}_{U F}$ in unbounded flows has proven so useful is that, even though it admits unphysical situations where particles may overlap, it is always symmetric, positive, and definite. ${ }^{11}$ We may occasionally reference the grand resistance tensor as well, but this is simply the inverse of the grand mobility tensor $\left(\mathcal{R}=\mathcal{M}^{-1}\right)$. We proceed to demonstrate how to incorporate a plane wall into the hydrodynamic interactions between colloidal particles so that these tensors are symmetric, positive, and definite.

Following the approach taken in the original Stokesian dynamics method, we consider the disturbance velocity, $\mathbf{u}^{\prime}(\mathbf{x})$, generated by a solid sphere of radius $a_{\beta}$ with its center at $\mathbf{x}_{\beta}$ a height $h_{\beta}$ above a plane wall with force density on its surface denoted $\mathbf{f}$. This disturbance field can be separated into two parts,

$$
\mathbf{u}^{\prime}(\mathbf{x})=\mathbf{u}^{i}(\mathbf{x})+\mathbf{u}^{w}(\mathbf{x}),
$$

where $\mathbf{u}^{i}(\mathbf{x})$ is the velocity field generated by an unbounded particle and $\mathbf{u}^{w}(\mathbf{x})$ is the reflection of the unbounded field off the plane wall such that the no-slip condition is satisfied,

$$
\mathbf{u}^{i}(\mathbf{x})+\mathbf{u}^{w}(\mathbf{x})=0
$$

when $\mathbf{x}$ is a point on the wall. We will need the Stokes flow Green's function, or Stokeslet, for the velocity field at $\mathbf{x}$ due to an isolated point force at $\mathbf{y}$,

$$
\mathbf{J}(\mathbf{x}, \mathbf{y})=\frac{1}{8 \pi \eta}\left(\frac{\mathbf{I}}{r}+\frac{\mathbf{r r}}{r^{3}}\right),
$$

where $\mathbf{I}$ is the identity tensor, $\mathbf{r}=\mathbf{x}-\mathbf{y}, r^{2}=\mathbf{r} \cdot \mathbf{r}$ and $\eta$ is the viscosity of the surrounding fluid. In addition, we need Blake's solution ${ }^{12}$ for the image of a Stokeslet above a wall located at $H$ in the fluid with normal $\boldsymbol{\delta}_{3}$,

$$
\begin{aligned}
\mathbf{J}^{w}(\mathbf{x}, \mathbf{y} ; H)= & -\mathbf{J}\left(\mathbf{x}, \mathbf{y}^{\prime}\right)+\left(y_{3}-H\right)^{2} \nabla_{y^{\prime}}^{2} \mathbf{J}\left(\mathbf{x}, \mathbf{y}^{\prime}\right) \cdot \mathbf{P} \\
& +2\left(y_{3}-H\right)\left(\mathbf{P} \cdot \nabla_{y^{\prime}} \mathbf{J}\left(\mathbf{x}, \mathbf{y}^{\prime}\right) \cdot \boldsymbol{\delta}_{3}\right)^{T},
\end{aligned}
$$

where $\mathbf{y}^{\prime}=\mathbf{y}-2\left(y_{3}-H\right) \boldsymbol{\delta}_{3}, \mathbf{P}=\mathbf{I}-2 \boldsymbol{\delta}_{3} \boldsymbol{\delta}_{3}$, and the superscript $T$ indicates transposition. With these we can write the exact solution for the disturbance velocity caused by particle $\beta$ as

$$
\mathbf{u}^{\prime}(\mathbf{x})=\int_{S_{\beta}} \mathbf{G}(\mathbf{x}, \mathbf{y} ; H) \cdot \mathbf{f}(\mathbf{y}) d S_{y},
$$

where the total Green's function is

$$
\mathbf{G}(\mathbf{x}, \mathbf{y} ; H)=\mathbf{J}(\mathbf{x}, \mathbf{y})+\mathbf{J}^{w}(\mathbf{x}, \mathbf{y} ; H) .
$$

From this exact expression we expand in surface moments the force density on particle $\beta$ following the Stokesian dynamics procedure to obtain

$$
\begin{aligned}
\mathbf{u}^{\prime}(\mathbf{x})= & \left.\left(1+\frac{a_{\beta}^{2}}{6} \nabla_{y}^{2}\right) \mathbf{G}(\mathbf{x}, \mathbf{y} ; H)\right|_{\mathbf{y}=\mathbf{x}_{\beta}} \cdot \mathbf{F}_{\beta} \\
& +\frac{1}{2} \nabla_{y} \times\left.\mathbf{G}(\mathbf{x}, \mathbf{y} ; H)\right|_{\mathbf{y}=\mathbf{x}_{\beta}} \cdot \mathbf{L}_{\beta} \\
& +\left.\left(1+\frac{a_{\beta}^{2}}{10} \nabla_{y}^{2}\right) \mathbf{K}(\mathbf{x}, \mathbf{y} ; H)\right|_{\mathbf{y}=\mathbf{x}_{\beta}}: \mathbf{S}_{\beta}+\cdots,
\end{aligned}
$$

where

$$
\mathbf{K}(\mathbf{x}, \mathbf{y} ; H)=\frac{1}{2}\left[\nabla_{y} \mathbf{G}(\mathbf{x}, \mathbf{y} ; H)+\left(\nabla_{y} \mathbf{G}(\mathbf{x}, \mathbf{y} ; H)\right)^{T}\right] .
$$

We choose to truncate these expressions at the stresslet level, though there is no reason that they cannot be expanded further to include higher order and faster decaying moments of the Green's function. The key step to obtaining the correct hydrodynamic interactions is to note that care must be used when taking the derivatives with respect to $\mathbf{y}$ of Blake's solution for the reflected velocity field, $\mathbf{J}^{w}(\mathbf{x}, \mathbf{y} ; H)$ depends explicitly on both $\mathbf{y}$ and $\mathbf{x}-\mathbf{y}^{\prime}$, reflecting the fact that the point force density $\mathbf{f}$ is distributed on the surface of the particle and not simply located at the particle's center. The correct derivatives are complex, but the following chain rules: 


$$
\begin{aligned}
\nabla_{y}\left(f\left(y_{3}\right) g(\mathbf{R})\right)= & \boldsymbol{\delta}_{3} f^{\prime}\left(y_{3}\right) g(\mathbf{R})-f\left(y_{3}\right) \mathbf{P} \cdot \nabla_{R} g(\mathbf{R}), \\
\nabla_{y}^{2}\left(f\left(y_{3}\right) g(\mathbf{R})\right)= & f^{\prime \prime}\left(y_{3}\right) g(\mathbf{R})+2 f^{\prime}\left(y_{3}\right) \boldsymbol{\delta}_{3} \cdot \nabla_{R} g(\mathbf{R}) \\
& +f\left(y_{3}\right) \nabla_{R}^{2} g(\mathbf{R}),
\end{aligned}
$$

where $\mathbf{R}=\mathbf{x}-\mathbf{y}^{\prime}$, allow for considerable simplification of this process.

We can also use these expressions to say something about the reflections of higher order Stokes flow singularities from a plane wall. From Eq. (8), we see that a spherical particle with a constant force density on its surface denoted $\mathbf{F}_{\beta} / 4 \pi a_{\beta}^{2}$, behaves as though it generates two singularities in the surrounding fluid at the particle's center. These singularities are the familiar Stokeslet and the source doublet $\left[\nabla_{y}^{2} \mathbf{J}(\mathbf{x}, \mathbf{y})\right]$. The same Fourier transform approach used to compute Blake's expression for the reflection of the Stokeslet $\left[\mathbf{J}^{w}(\mathbf{x}, \mathbf{y} ; H)\right]$ can be used to compute the reflection of the source doublet. ${ }^{13}$ While this procedure is arduous, the resulting reflection of the source doublet could have been used to generate the reflected field $\mathbf{u}^{w}(\mathbf{x})$ directly. Knowing that the Stokes equations are unique, we can see from Eq. (8) that the reflected field has only two contributions: Blake's reflection of the Stokeslet and one other which must be the reflection of the source doublet. This suggests a direct and facile way to compute reflections of higher order Stokes flow singularities, something which is quite subtle and has recently been a source of confusion. ${ }^{14}$ This approach has been used in similar circumstances, ${ }^{15}$ but we state it here explicitly since it applies to any reflection of a Stokes flow singularity. Namely, no complicated Fourier transform or limiting process is needed to compute these reflections. One simply writes the higher order singularity as a derivative of the Stokeslet or other fundamental singularity with a given condition on the wall, and the reflection of that singularity is simply the same derivative (with respect to the source point, $\mathbf{y}$ ) of Blake's reflection or fundamental singularity. That is, we can simply write the no slip condition on the wall as

$$
\nabla_{y}^{n} \mathbf{J}(\mathbf{x}, \mathbf{y})+\nabla_{y}^{n} \mathbf{J}^{w}(\mathbf{x}, \mathbf{y} ; H)=0
$$

when $\mathbf{x}$ is a point on the plane wall $\left(x_{3}=H\right)$. This also suggests one way to simulate the hydrodynamics of particles constrained by more than one wall or some curvilinear geometry. If we know the reflection of the Stokeslet satisfying the boundary conditions on the wall, then we can compute the disturbance velocity generated by a particle with an arbitrary force density using the procedure just described.

The mobility tensor is constructed from the disturbance velocity field $\mathbf{u}^{\prime}(\mathbf{x})$ and Faxén formulas for a particle near a wall, which, unfortunately are not known. However, we can bypass the need to know the exact Faxén formulas with a wall by noting that the flow caused by the reflection from the wall $\mathbf{u}^{w}(\mathbf{x})$ is just another disturbance flow and has no singularities within the fluid region above the wall. Therefore, the usual, well-known ${ }^{9}$ Faxén formulas can be applied. That is, the Faxén formulas coupling a disturbance field in the fluid to the translational and rotational velocities of a spherical particle $(\alpha)$ of radius $a_{\alpha}$ centered at $\mathbf{x}_{\alpha}$ relative to the fluid $\left(\mathbf{U}_{\alpha}-\mathbf{U}_{\alpha}^{\infty}, \boldsymbol{\Omega}_{\alpha}-\boldsymbol{\Omega}_{\alpha}^{\infty}\right)$ and the rate of strain of the fluid $\left(\mathbf{E}_{\alpha}^{\infty}\right)$ are

$$
\begin{aligned}
& \mathbf{U}_{\alpha}-\mathbf{U}_{\alpha}^{\infty}=\frac{\mathbf{F}_{\alpha}}{6 \pi \eta a_{\alpha}}+\left.\left(1+\frac{a_{\alpha}^{2}}{6} \nabla_{x}^{2}\right) \mathbf{u}^{\prime}(\mathbf{x})\right|_{\mathbf{x}_{\alpha}}, \\
& \Omega_{\alpha}-\Omega_{\alpha}^{\infty}=\frac{\mathbf{L}_{\alpha}}{8 \pi \eta a_{\alpha}^{3}}+\frac{1}{2} \nabla_{x} \times\left.\mathbf{u}^{\prime}(\mathbf{x})\right|_{\mathbf{x}_{\alpha}}, \\
& -\mathbf{E}_{\alpha}^{\infty}=\frac{\mathbf{S}_{\alpha}}{\frac{20}{3} \pi \eta a_{\alpha}^{3}}+\left.\left(1+\frac{a_{\alpha}^{2}}{10} \nabla_{x}^{2}\right) \mathbf{e}^{\prime}(\mathbf{x})\right|_{\mathbf{x}_{\alpha}},
\end{aligned}
$$

where the disturbance rate of strain is

$$
\mathbf{e}^{\prime}(\mathbf{x})=\frac{1}{2}\left[\nabla_{x} \mathbf{u}^{\prime}(\mathbf{x})+\left(\nabla_{x} \mathbf{u}^{\prime}(\mathbf{x})\right)^{T}\right] .
$$

In principle, since we have a detailed knowledge of the disturbance field caused by particle $\beta$, we can compute how it interacts hydrodynamically with particle $\alpha$. For that matter, if $\alpha$ and $\beta$ are the same particle, we can compute the hydrodynamic interaction between just a single particle and a wall by only considering the reflected part of the disturbance field, $\mathbf{u}^{w}(\mathbf{x})$.

In order to complete our description of the hydrodynamic interactions, we need to compute the mobility and resistance tensors. Each term in the grand mobility tensor is constructed from the combination of a Faxén formula with the singularities from a force multipole in the disturbance velocity field. As an example, we construct the term $\mathbf{M}_{U F}$ explicitly using Eqs. (8) and (12) to characterize the coupling of a force on particle $\beta$ to the relative velocity of particle $\alpha$,

$$
\mathbf{M}_{U F}^{\alpha \beta}=\left.\left(1+\frac{a_{\alpha}^{2}}{6} \nabla_{x}^{2}\right)\left(1+\frac{a_{\beta}^{2}}{6} \nabla_{y}^{2}\right) \mathbf{G}(\mathbf{x}, \mathbf{y} ; H)\right|_{\mathbf{x}=\mathbf{x}_{\alpha}} ^{\mathbf{y}=\mathbf{x}_{\beta}} .
$$

The other grand mobility tensor terms are constructed analogously. One additional note is necessary, however. For the coupling between particle $\alpha$ and itself, we discard the disturbance velocities generated by the Stokeslet and use the reflected field alone in the Faxén formulas. This is illustrated for the term $\mathbf{M}_{U F}^{\alpha \alpha}$,

$\mathbf{M}_{U F}^{\alpha \alpha}=\frac{\mathbf{I}}{6 \pi \eta a_{\alpha}}+\left.\left(1+\frac{a_{\alpha}^{2}}{6} \nabla_{x}^{2}\right)\left(1+\frac{a_{\alpha}^{2}}{6} \nabla_{y}^{2}\right) \mathbf{J}^{w}(\mathbf{x}, \mathbf{y} ; H)\right|_{\mathbf{x}=\mathbf{x}_{\alpha}} ^{\mathbf{y}=\mathbf{x}_{\alpha}}$.

All terms in the mobility tensor are given explicitly in Appendices A-C.

The grand resistance tensor, denoted by

$$
\mathcal{R}=\left(\begin{array}{cccc}
\mathbf{R}_{F U} & \mathbf{R}_{F \Omega} & \mathbf{R}_{F E} & \ldots \\
\mathbf{R}_{L U} & \mathbf{R}_{L \Omega} & \mathbf{R}_{L E} & \ldots \\
\mathbf{R}_{S U} & \mathbf{R}_{S \Omega} & \mathbf{R}_{S E} & \ldots \\
\vdots & \vdots & \vdots & \ddots
\end{array}\right),
$$

is simply the inverse of the grand mobility tensor. However, since we truncate the expansion generating the grand mobility tensor after a finite number of multipoles, this tensor is only a far-field approximation. We wish to include lubrica- 
tion interactions which are only properly expressed after including a large (infinite) number of force multipoles. After inverting the fully truncated grand mobility tensor, we add in the exact lubrication forms for the resistance tensor for both the interactions between near pairs of particles ${ }^{9}\left(\mathcal{R}^{P}\right)$ and a particle near the wall ${ }^{5}\left(\mathcal{R}^{W}\right)$. In addition to this, we subtract out the far-field contributions arising from the inversion of the grand mobility tensor $\left(\mathcal{R}^{P, \infty}+\mathcal{R}^{W, \infty}\right)$ to avoid over counting the hydrodynamic interactions. The grand resistance tensor, $\mathcal{R}$, including these lubrication contributions is

$$
\mathcal{R}=\mathcal{M}^{-1}+\mathcal{R}^{P}+\mathcal{R}^{W}-\left(\mathcal{R}^{P, \infty}+\mathcal{R}^{W, \infty}\right) .
$$

Elements of this resistance tensor can now be used in the Langevin equation for particle dynamics, correctly including the far-field, many-body hydrodynamic interactions and the singular, pairwise lubrication forces, and is symmetric positive-definite by construction. The details of how this is done can be found in the thorough discussion by Phung, Brady, and Bossis. ${ }^{16}$

\section{RESULTS AND DISCUSSION}

\section{A. Symmetric mobility and resistance tensors}

The above approach follows the well-established Stokesian dynamics procedure for constructing a symmetric, positive-definite mobility tensor, and so it is somewhat surprising that two prior studies of the problem of spherical particles moving in Stokes flow near a plane wall failed to compute symmetric mobility and resistance tensors. The first approach by Bossis, Meunier, and Sherwood ${ }^{5}$ used a technique similar to the one discussed above in which they expand the reflection of the Stokeslet in force multipoles to derive the wall contribution to the mobility tensor. However, they did not appreciate that the reflection of the Stokeslet is a function of how far the point force is from the wall as well as the separation vector $\mathbf{x}-\mathbf{y}^{\prime}$. We take care to write the reflection as $\mathbf{J}^{w}(\mathbf{x}, \mathbf{y} ; H)$ which is an explicit function of a destination, a source, and the location of the wall. Their multipole expansion presumes a dependence on $\mathbf{x}-\mathbf{y}^{\prime}$ only and as a result, the mobility tensors stemming from this approach are not symmetric. In a quantitative sense, this error may not be large; however, their approach is unusable in the context of Brownian motion which requires a symmetric mobility tensor. Similarly, in their study of confined chains of Brownian particles, Jendrejack et $a l^{6}{ }^{6}$ numerically compute the reflection off a wall of the Rotne-Prager tensor. Recall that the R-P tensor is simply $\mathbf{J}^{R}(\mathbf{r})=\left(1+a^{2} / 3 \nabla_{r}^{2}\right) \mathbf{J}(\mathbf{r})$. They call this reflection the contribution to the mobility tensor due to the wall, but do not recognize that the Rotne-Prager tensor lacks a direct connection with the fluid velocity field surrounding the particle. It cannot be used to generate the reflection off the wall since it depends on a linear combination of the disturbance velocity generated by an isolated particle and its source doublet and not just the disturbance velocity itself. As shown earlier, the reflected velocity field is still subject to the Faxén formulas which produces extra quadrupolar and octupolar contributions to the mobility that are essential to maintaining symmetry. Again this method is unsuitable for simulating Brownian particles. Jendrejack et al. recognized that
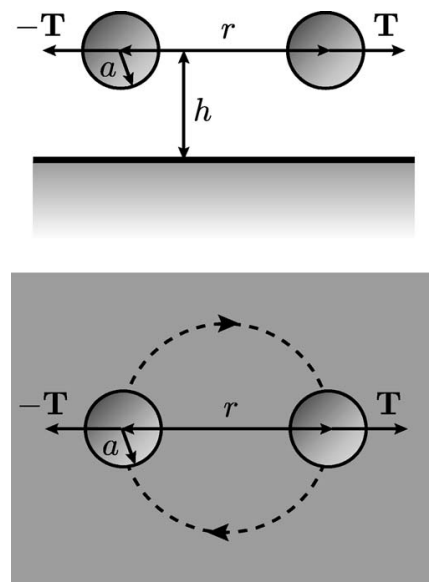

FIG. 2. A side-on and top-down view of a pair of particles above a plane wall with equal and opposite torques $T$ applied along their line of centers. The particles have the same radius $a$, are separated by a distance $r$, and are a height $h$ above the wall.

their mobility tensor was not symmetric and simply symmetrized it by adding the transpose and dividing by two to conduct Brownian simulations. There is, unfortunately, no way to assess to accuracy of this manipulation.

\section{B. Co-rotation of a doublet of particles}

Illustrated in Fig. 2 is a pair of spherical particles of the same radius with equal and opposite torques applied along their line of centers. One can imagine accomplishing this by connecting a pair of spherical particles with a slender torsion wire and twisting those particles to load a torque into the wire. For a pair of particles in an unbounded fluid, the torque along the line of centers causes the particles to rotate about their line of centers in opposite directions while remaining otherwise still. However, when the doublet is brought near a wall, the particles both rotate and translate because the wall induces an additional coupling between torque and translation. Since these particles might be connected by a wire, the separation between the particles remains the same and the doublet spins about its center of mass. We can measure the tensile force on the wire as the doublet rotates, but one can show that because the grand mobility tensor is symmetric, this tensile force is exactly zero regardless of separation and height above the wall. In a model lacking symmetry, some nonzero force along the line of centers is necessary to maintain the separation between the particles. Interestingly, as indicated in Fig. 3, the rate of rotation of the doublet about its center of mass, $\Omega$, normalized by the torque on the particles, $T / 8 \pi \eta a^{3}$, is a nonmonotonic function of both the separation between the particles, $r$, and the height of the doublet above the wall, $h$. When the particles are far apart, the rate of rotation of the doublet is decreasing as $r$ increases because the translational speed of a particle in the doublet $(\Omega r / 2)$ is set only by the coupling of a single particle to the wall. When the particles are far from the wall, the rate of rotation of the doublet is also decreasing as $h$ increases. However this is caused by weakening interactions with the wall. Conversely, when the separation between the particles becomes quite small or the doublet is close to the wall, the rotation rate of 


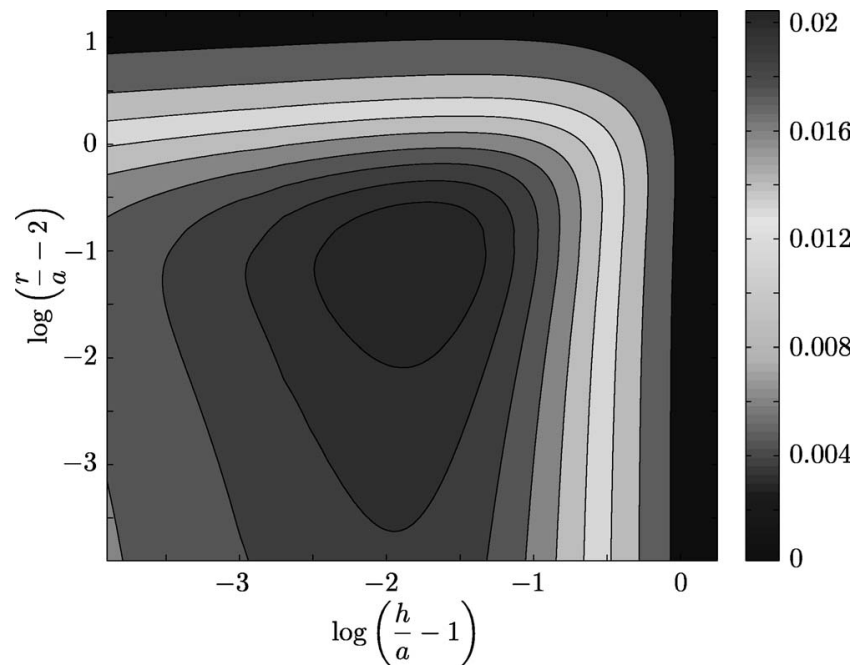

FIG. 3. The rotation rate of the doublet about its center of mass normalized by the torque on the particles about their line of centers $\left(8 \pi \eta a^{3} \Omega / T\right)$. The normalized rate of rotation is maximum near $r=2.1 a, h=1.01 a$.

the doublet also decreases because in this limit the resistance to motion of the particles becomes singular. Since the normalized rotation rate is decreasing in the limits that the particles are both near and far apart and the doublet is both near and far from the wall, it must reach a global maximum where the doublet rotates most quickly.

Since the torques on the particles are equal and opposite, the doublet is a force and torque free object. It can be thought of as a model for a number of different interesting systems. A recent study of bacteria swimming near a wall found that the head and flagellum of the organism co-rotate just like this doublet and for the exact same reason. ${ }^{10} \mathrm{~A}$ swimming bacterium is a force and torque free object and in order to propel itself by torquing its flagellum it must also have an equal and opposite torque on its head. The study found that the same coupling to the wall that cause the doublet to co-rotate also cause a bacterium to sweep out arcs as it swims near a wall rather than swim straight. The only difference between the doublet and the bacterium is that the bacterium is also propelled along its line of centers.

\section{Grand mobility tensors for any confining boundaries}

The procedure described in Sec. II is hardly limited to the single plane wall geometry. For any confining geometry, we can write the disturbance velocity field at $\mathbf{x}$ generated by a point force at $\mathbf{y}$ as

$$
\begin{aligned}
\mathbf{u}(\mathbf{x})= & \mathbf{G}\left(\mathbf{x}, \mathbf{y} ; H_{1}, H_{2}, \ldots\right) \cdot \mathbf{f}(\mathbf{y})=\mathbf{J}(\mathbf{x}, \mathbf{y}) \cdot \mathbf{f}(\mathbf{y}) \\
& +\mathbf{J}^{w}\left(\mathbf{x}, \mathbf{y} ; H_{1}, H_{2}, \ldots\right) \cdot \mathbf{f}(\mathbf{y}),
\end{aligned}
$$

where $\mathbf{J}^{w}\left(\mathbf{x}, \mathbf{y} ; H_{1}, H_{2}, \ldots\right)$ is the reflection of the Stokeslet off the confining surfaces and $H_{1}, H_{2}, \ldots$ are just geometrical parameters. It is clear that the terms of the mobility tensor are constructed in exactly the same way as for a single plane wall. No changes in procedure are necessary since the process only relies on properties of the Stokes equations and not on the geometrical constraints.
As a specific example, consider particles in a fluid bounded by a flat and nondeformable free surface; the fluid is constrained only by a no-penetration condition at the surface. The Green's function in this case is similar to the plane wall Green's function. It is the sum of a Stokeslet above the interface and a stokeslet with a reflected force density below the interface, viz.,

$$
\mathbf{G}(\mathbf{x}, \mathbf{y} ; H)=\mathbf{J}(\mathbf{x}, \mathbf{y})+\mathbf{J}\left(\mathbf{x}, \mathbf{y}^{\prime}\right) \cdot \mathbf{P} .
$$

Nothing about this Green's function precludes using Eqs. (16) and (17) and their analogs to build up the grand mobility tensor. In fact, the same caveats for taking the derivatives with respect to the source, $\mathbf{y}$, apply to this Green's function too. A clever implementation of a traditional Stokesian dynamics simulation can model this interface as well, by recognizing that a particle near a free surface acts as though there were an image particle with a reflected force density on its surface below the interface, one can simulate $N$ particles near a free surface using those $N$ particles and $N$ image particles. The extra particles increase the computational time for conventional Stokesian dynamics by a factor of 8 over that for $N$ unbounded particles. Using the method described herein with the Green's function in Eq. (21), that multiplicative factor is near unity.

It is worth noting further that the grand mobility tensor for particles near interfaces with even more complicated boundary conditions may be constructed by using this method directly or by using the linear combination of mobility tensors with simpler confinements. Consider particles moving near a flat and nondeformable liquid-liquid interface where the ratio of the viscosity of the confining liquid to the viscosity of the embedding liquid is $\lambda$. Constructing a simulation using image particles as in the free surface problem is now quite difficult. However, the Green's function in this case is a linear combination of the Green's functions for a free surface $\left[\mathbf{G}^{F}(\mathbf{x}, \mathbf{y} ; H)\right.$, Eq. (21)] and a solid plane wall $\left[\mathbf{G}^{W}(\mathbf{x}, \mathbf{y} ; H)\right.$, Eq. (7)]

$$
\mathbf{G}(\mathbf{x}, \mathbf{y} ; H)=\left(\frac{1}{1+\lambda}\right) \mathbf{G}^{F}(\mathbf{x}, \mathbf{y} ; H)+\left(\frac{\lambda}{1+\lambda}\right) \mathbf{G}^{W}(\mathbf{x}, \mathbf{y} ; H),
$$

so that changing $\lambda$ from zero to infinity transitions from the free surface boundary to the plane wall boundary. ${ }^{17}$ Taking this same linear combination of the grand mobility tensors for particles near a free surface and particles near a plane wall generates the grand mobility tensor for particles near a liquid-liquid interface. As before, a resistance tensor may be constructed from the mobility tensor, where now one needs the resistance interactions for a single particle adjacent to a free surface or a liquid-liquid interface.

\section{CONCLUSIONS}

We have constructed a symmetric, positive-definite mobility tensor for particles near a plane wall. Additionally, we have shown how to compute a symmetric, positive-definite mobility tensor for particles in any confining geometry given 
the reflection of the Stokeslet off the confining walls. Interestingly, the scaling of the Stokesian dynamics algorithm with respect to the number of particles is independent of any confining geometry and only a few more computations per particle pair are necessary to build up the resistance and mobility tensors. While the expressions for the elements of the grand mobility terms in Appendices A-C are algebraically complicated, they may be tabulated and used with efficient table lookup procedures for dynamic simulation. Following this approach, one can now use Stokesian dynamics to study the behavior of suspensions of spherical particles in arbitrary confining geometries. In an even simpler approach, one may approximate certain confinements such as the parallel plate geometry by superimposing the mobility tensors due to a pair of plane walls with opposing normals. There are some indications that this approximation can be quite accurate, ${ }^{18}$ though it does trade the convenience of superposition for the fidelity of the exact two wall reflection.

Symmetry and positive-definiteness of the mobility tensor are fundamental to the hydrodynamic interactions between particles in Stokes flow and are essential to simulating Brownian motion. We have chosen to study the static properties of particle pairs here, illustrating the importance of symmetry in the physically interesting problem of a force and torque free doublet rotating above a plane wall. The Brownian motion of particles may be generated directly from the tensors derived herein, and can be used to study various dynamic processes of Brownian particles near a plane wall including self-assembly and colloidal crystallization using these tensors in Stokesian dynamics simulations.

\section{ACKNOWLEDGMENTS}

This work was supported in part by NSF Grant No. CBET 0506701.

\section{APPENDIX A: REFLECTED STOKESLET CONTRIBUTIONS TO THE GRAND MOBILITY TENSOR}

As in Eq. (16), we can write explicit expressions for all of the terms in the mobility tensor. For the purposes of this Appendix we only concern ourselves with the contributions due to reflection of the Stokeslet. The interactions due to the Stokeslet itself yield the well-known far-field grand mobility tensor for unbounded pairs of particles and can be found in Ref. 4. We write the contribution to the grand mobility matrix due to the reflected field, $\mathbf{J}^{w}(\mathbf{x}, \mathbf{y} ; H)$, as $\hat{\mathcal{M}}$, and the contributions to the subtensors are denoted $\hat{\mathbf{M}}_{U F}, \hat{\mathbf{M}}_{U L}, \ldots$ When this is added to the grand mobility tensor for an unbounded set of particles, we recover the complete grand mobility tensor, $\mathcal{M}$. Without loss of generality, these expressions can be used to generate the single particle-wall and particle pair-wall interactions as described in Sec. II. Note that we define the operator $\nabla_{x}^{T}$ such that $\nabla_{x}^{T} \mathbf{u}(\mathbf{x})=\left(\nabla_{x} \mathbf{u}(\mathbf{x})\right)^{T}$,

$$
\begin{aligned}
& \hat{\mathbf{M}}_{U F}^{\alpha \beta}=\left.\left(1+\frac{a_{\alpha}^{2}}{6} \nabla_{x}^{2}\right)\left(1+\frac{a_{\beta}^{2}}{6} \nabla_{y}^{2}\right) \mathbf{J}^{w}(\mathbf{x}, \mathbf{y} ; H)\right|_{\mathbf{x}=\mathbf{x}_{\alpha}} ^{\mathbf{y}=\mathbf{x}_{\beta}}, \\
& \hat{\mathbf{M}}_{\Omega F}^{\alpha \beta}=\frac{1}{2} \nabla_{x} \times\left.\left(1+\frac{a_{\beta}^{2}}{6} \nabla_{y}^{2}\right) \mathbf{J}^{w}(\mathbf{x}, \mathbf{y} ; H)\right|_{\mathbf{x}=\mathbf{x}_{\alpha}} ^{\mathbf{y}=\mathbf{x}_{\beta}}, \\
& \hat{\mathbf{M}}_{E F}^{\alpha \beta}=\left.\frac{1}{2}\left(\nabla_{x}+\nabla_{x}^{T}\right)\left(1+\frac{a_{\alpha}^{2}}{10} \nabla_{x}^{2}\right)\left(1+\frac{a_{\beta}^{2}}{6} \nabla_{y}^{2}\right) \mathbf{J}^{w}(\mathbf{x}, \mathbf{y} ; H)\right|_{\mathbf{x}=\mathbf{x}_{\alpha}} ^{\mathbf{y}=\mathbf{x}_{\beta}}, \\
& \hat{\mathbf{M}}_{U L}^{\alpha \beta}=\left(1+\frac{a_{\alpha}^{2}}{6} \nabla_{x}^{2}\right) \frac{1}{2} \nabla_{y} \times\left.\mathbf{J}^{w}(\mathbf{x}, \mathbf{y} ; H)\right|_{\mathbf{x}=\mathbf{x}_{\alpha}} ^{\mathbf{y}=\mathbf{x}_{\beta}}, \\
& \hat{\mathbf{M}}_{\Omega L}^{\alpha \beta}=\frac{1}{2} \nabla_{x} \times \frac{1}{2} \nabla_{y} \times\left.\mathbf{J}^{w}(\mathbf{x}, \mathbf{y} ; H)\right|_{\mathbf{x}=\mathbf{x}_{\alpha}} ^{\mathbf{y}=\mathbf{x}_{\beta}}, \\
& \hat{\mathbf{M}}_{E L}^{\alpha \beta}=\frac{1}{2}\left(\nabla_{x}+\nabla_{x}^{T}\right)\left(1+\frac{a_{\alpha}^{2}}{10} \nabla_{x}^{2}\right) \frac{1}{2} \nabla_{y} \times\left.\mathbf{J}^{w}(\mathbf{x}, \mathbf{y} ; H)\right|_{\mathbf{x}=\mathbf{x}_{\alpha}} ^{\mathbf{y}=\mathbf{x}_{\beta}}, \\
& \hat{\mathbf{M}}_{U S}^{\alpha \beta}=\left.\left(1+\frac{a_{\alpha}^{2}}{6} \nabla_{x}^{2}\right)\left(1+\frac{a_{\beta}^{2}}{10} \nabla_{y}^{2}\right) \mathbf{K}^{w}(\mathbf{x}, \mathbf{y} ; H)\right|_{\mathbf{x}=\mathbf{x}_{\alpha}} ^{\mathbf{y}=\mathbf{x}_{\beta}}, \\
& \hat{\mathbf{M}}_{\Omega S}^{\alpha \beta}=\frac{1}{2} \nabla_{x} \times\left.\left(1+\frac{a_{\beta}^{2}}{10} \nabla_{y}^{2}\right) \mathbf{K}^{w}(\mathbf{x}, \mathbf{y} ; H)\right|_{\mathbf{x}=\mathbf{x}_{\alpha}} ^{\mathbf{y}=\mathbf{x}_{\beta}}, \\
& \hat{\mathbf{M}}_{E S}^{\alpha \beta}=\left.\frac{1}{2}\left(\nabla_{x}+\nabla_{x}^{T}\right)\left(1+\frac{a_{\alpha}^{2}}{10} \nabla_{x}^{2}\right)\left(1+\frac{a_{\beta}^{2}}{10} \nabla_{y}^{2}\right) \mathbf{K}^{w}(\mathbf{x}, \mathbf{y} ; H)\right|_{\mathbf{x}=\mathbf{x}_{\alpha}} ^{\mathbf{y}=\mathbf{x}_{\beta}} .
\end{aligned}
$$

\section{APPENDIX B: PARTICLE-WALL “SELF” MOBILITY TENSOR $(\boldsymbol{\alpha} \alpha)$}

Presented below is the contribution to the grand mobility tensor due to a single particle $(\alpha)$ interacting with a plane wall. All of the following terms are normalized by $6 \pi \eta a_{\alpha}^{n}$, where $n$ is chosen to keep things dimensionally consistent. Additionally, $h$ is the normalized height of the particle above the wall such that $h=h_{\alpha} / a_{\alpha}$. Note that since the mobility tensor is symmetric by construction, we include only six of nine subtensors. The other three can be computed directly taking a transposition, 


$$
\begin{aligned}
\hat{M}_{U F, i j}^{\alpha \alpha}= & -\frac{1}{16}\left(9 h^{-1}-2 h^{-3}+h^{-5}\right)\left(\delta_{i j}-\delta_{i 3} \delta_{j 3}\right) \\
& -\frac{1}{8}\left(9 h^{-1}-4 h^{-3}+h^{-5}\right) \delta_{i 3} \delta_{j 3}, \\
\hat{M}_{\Omega F, i j}^{\alpha \alpha}= & \frac{3}{32} h^{-4} \epsilon_{3 i j}, \\
\hat{M}_{\Omega L, i j}^{\alpha \alpha}= & -\frac{15}{64} h^{-3}\left(\delta_{i j}-\delta_{i 3} \delta_{j 3}\right)-\frac{3}{32} h^{-3} \delta_{i 3} \delta_{j 3},
\end{aligned}
$$

$$
\begin{aligned}
\hat{M}_{E F, i j k}^{\alpha \alpha}= & -\frac{3}{160}\left(15 h^{-2}-12 h^{-4}+5 h^{-6}\right)\left[\left(\delta_{i k}-\delta_{i 3} \delta_{k 3}\right) \delta_{j 3}\right. \\
& \left.+\left(\delta_{j k}-\delta_{j 3} \delta_{k 3}\right) \delta_{i 3}\right]+\frac{3}{32}\left(3 h^{-2}-3 h^{-4}+h^{-6}\right) \\
& \times\left(\delta_{i j}-\delta_{i 3} \delta_{j 3}\right) \delta_{k 3}-\frac{3}{16}\left(3 h^{-2}-3 h^{-4}+h^{-6}\right) \\
& \times \delta_{i 3} \delta_{j 3} \delta_{k 3}, \\
\hat{M}_{E L, i j k}^{\alpha \alpha}= & -\frac{9}{320}\left(5 h^{-3}-4 h^{-5}\right)\left(\delta_{j 3} \epsilon_{3 i k}+\delta_{i 3} \epsilon_{3 j k}\right),
\end{aligned}
$$

$$
\begin{aligned}
\hat{M}_{E S, i j k l}^{\alpha \alpha}= & -\frac{3}{640}\left(10 h^{-3}-24 h^{-5}+9 h^{-7}\right)\left(\delta_{i j}-\delta_{i 3} \delta_{j 3}\right)\left(\delta_{k l}-\delta_{k 3} \delta_{l 3}\right)-\frac{9}{640}\left(10 h^{-3}-8 h^{-5}+3 h^{-7}\right)\left[\left(\delta_{i k}-\delta_{i 3} \delta_{k 3}\right)\left(\delta_{j l}-\delta_{j 3} \delta_{l 3}\right)\right. \\
& \left.+\left(\delta_{i l}-\delta_{i 3} \delta_{l 3}\right)\left(\delta_{j k}-\delta_{j 3} \delta_{k 3}\right)\right]+\frac{3}{160}\left(20 h^{-3}-24 h^{-5}+9 h^{-7}\right)\left[\left(\delta_{i j}-\delta_{i 3} \delta_{j 3}\right) \delta_{k 3} \delta_{l 3}+\left(\delta_{k l}-\delta_{k 3} \delta_{l 3}\right) \delta_{i 3} \delta_{j 3}\right] \\
& -\frac{9}{320}\left(15 h^{-3}-16 h^{-5}+6 h^{-7}\right)\left[\left(\delta_{i k}-\delta_{i 3} \delta_{k 3}\right) \delta_{j 3} \delta_{l 3}+\left(\delta_{i l}-\delta_{i 3} \delta_{l 3}\right) \delta_{j 3} \delta_{k 3}+\left(\delta_{j k}-\delta_{j 3} \delta_{k 3}\right) \delta_{i 3} \delta_{l 3}+\left(\delta_{j l}-\delta_{j 3} \delta_{l 3}\right) \delta_{i 3} \delta_{k 3}\right] \\
& -\frac{3}{80}\left(20 h^{-3}-24 h^{-5}+9 h^{-7}\right) \delta_{i 3} \delta_{j 3} \delta_{k 3} \delta_{l 3} .
\end{aligned}
$$

\section{APPENDIX C: PARTICLE-WALL "PAIR” MOBILITY TENSOR $(\boldsymbol{\alpha} \boldsymbol{\beta})$}

Here we present the contributions to the grand mobility tensor due to interactions between an identically sized particle pair $(\alpha \beta)$ and a plane wall. One can generate these tensors for particles of different sizes just as easily using the expressions in Appendix A. However, the expressions for those tensors are significantly longer. As above, the mobility terms are normalized by $6 \pi \eta a^{n}$, where $a=a_{\alpha}=a_{\beta}$ and $n$ is selected to provide the correct dimensionality. We define the following as well:

$$
\mathbf{R}=\frac{1}{a}\left(\mathbf{x}_{\alpha}-\mathbf{x}_{\beta}+2 h_{\beta} \boldsymbol{\delta}_{3}\right)
$$

and $\mathbf{e}=\mathbf{R} / R$, where $R=\sqrt{\mathbf{R} \cdot \mathbf{R}}$. Additionally, we define a rescaled height above the wall as $\hat{h}=h_{\beta} /\left(a R_{3}\right)$. As in the previous section we include six of the nine subtensors. The others may be generated through transposition,

$$
\begin{aligned}
\hat{M}_{U F, i j}^{\alpha \beta}= & -\frac{1}{4}\left[3\left(1+2 \hat{h}(1-\hat{h}) e_{3}^{2}\right) R^{-1}+2\left(1-3 e_{3}^{2}\right) R^{-3}-2\left(1-5 e_{3}^{2}\right) R^{-5}\right] \delta_{i j}-\frac{1}{4}\left[3\left(1-6 \hat{h}(1-\hat{h}) e_{3}^{2}\right) R^{-1}-6\left(1-5 e_{3}^{2}\right) R^{-3}\right. \\
& \left.+10\left(1-7 e_{3}^{2}\right) R^{-5}\right] e_{i} e_{j}+\frac{1}{2} e_{3}\left[3 \hat{h}\left(1-6(1-\hat{h}) e_{3}^{2}\right) R^{-1}-6\left(1-5 e_{3}^{2}\right) R^{-3}+10\left(2-7 e_{3}^{2}\right) R^{-5}\right] e_{i} \delta_{j 3} \\
& +\frac{1}{2} e_{3}\left(3 \hat{h} R^{-1}-10 R^{-5}\right) \delta_{i 3} e_{j}-\left[3 \hat{h}^{2} e_{3}^{2} R^{-1}+3 e_{3}^{2} R^{-3}+\left(2-15 e_{3}^{2}\right) R^{-5}\right] \delta_{i 3} \delta_{j 3}, \\
\hat{M}_{\Omega F, i j}^{\alpha \beta}= & \frac{3}{4} R^{-2} \epsilon_{i j k} e_{k}+\frac{3}{2}\left[6 \hat{h}_{3}^{2} R^{-2}+\left(1-10 e_{3}^{2}\right) R^{-4}\right] \epsilon_{3 k i} e_{k} \delta_{j 3}-\frac{3}{2} e_{3}\left(3 \hat{h} R^{-2}-5 R^{-4}\right) \epsilon_{3 k i} e_{k} e_{j}-\frac{3}{2} e_{3}\left(\hat{h} R^{-2}-R^{-4}\right) \epsilon_{3 i j}, \\
\hat{M}_{\Omega L, i j}^{\alpha \beta}= & \frac{3}{8}\left(1-6 e_{3}^{2}\right) R^{-3} \delta_{i j}-\frac{9}{8} R^{-3} e_{i} e_{j}+\frac{9}{4} e_{3} R^{-3} \delta_{i 3} e_{j}+\frac{9}{4} R^{-3} \epsilon_{3 k i} \epsilon_{3 l j} e_{k} e_{l}, \\
\hat{M}_{E F, i j k}^{\alpha \beta}= & \frac{6}{5} e_{3}\left[(5-2 \hat{h}) R^{-4}-15 R^{-6}\right] \delta_{i 3} \delta_{j 3} \delta_{k 3}+3 R^{-6} \delta_{i 3} \delta_{j 3} e_{k}-\frac{3}{10} e_{3}\left[5 \hat{h}\left(1-6(1-\hat{h}) e_{3}^{2}\right) R^{-2}-2\left(5-\hat{h}-5(5-2 \hat{h}) e_{3}^{2}\right) R^{-4}\right. \\
& \left.+10\left(2-7 e_{3}^{2}\right) R^{-6}\right] \delta_{i j} \delta_{k 3}-\frac{3}{10}\left[(5-2 \hat{h}) R^{-4}-10 R^{-6}\right] e_{3}\left(\delta_{i k} \delta_{j 3}+\delta_{i 3} \delta_{j k}\right)+\frac{3}{2} e_{3}\left[(5-2 \hat{h}) R^{-4}-14 R^{-6}\right]\left(\delta_{j 3} e_{i} e_{k}+\delta_{i 3} e_{j} e_{k}\right) \\
& +\frac{3}{2}\left[6 \hat{h}(1-\hat{h}) e_{3}^{2} R^{-2}+\left(1-4(5-2 \hat{h}) e_{3}^{2}\right) R^{-4}-2\left(2-21 e_{3}^{2}\right) R^{-6}\right]\left(e_{i} \delta_{j 3} \delta_{k 3}+e_{j} \delta_{i 3} \delta_{k 3}\right)-\frac{3}{10}\left[15 \hat{h}(1-\hat{h}) e_{3}^{2} R^{-2}\right. \\
& \left.+\left(4-5(5-2 \hat{h}) e_{3}^{2}\right) R^{-4}-5\left(1-7 e_{3}^{2}\right) R^{-6}\right]\left(e_{i} \delta_{j k}+e_{j} \delta_{i k}\right)+\frac{3}{2} e_{3}\left[3 \hat{h}\left(1-10(1-\hat{h}) e_{3}^{2}\right) R^{-2}-2\left(5-\hat{h}-7(5-2 \hat{h}) e_{3}^{2}\right) R^{-4}\right. \\
& \left.+14\left(2-9 e_{3}^{2}\right) R^{-6}\right] e_{i} e_{j} \delta_{k 3}+\frac{3}{20}\left[5\left(1-6 \hat{h}(1-\hat{h}) e_{3}^{2}\right) R^{-2}-2\left(4-5(5-2 \hat{h}) e_{3}^{2}\right) R^{-4}+10\left(1-7 e_{3}^{2}\right) R^{-6}\right] \delta_{i j} e_{k} \\
& -\frac{3}{4}\left[3\left(1-10(1-\hat{h}) e_{3}^{2}\right) R^{-2}-2\left(4-7(5-2 \hat{h}) e_{3}^{2}\right) R^{-4}-14\left(1-9 e_{3}^{2}\right) R^{-6}\right] e_{i} e_{j} e_{k},
\end{aligned}
$$




$$
\begin{aligned}
& \hat{M}_{E L, i j k}^{\alpha \beta}=-\frac{9}{8} R^{-3}\left(e_{j} \epsilon_{i k l} e_{l}+e_{i} \epsilon_{j k l} e_{l}\right)-\frac{9}{10} R^{-5}\left(\delta_{j 3} \epsilon_{3 i k}+\delta_{i 3} \epsilon_{3 j k}\right)+\frac{9}{4}\left(R^{-3}-2 R^{-5}\right)\left(e_{i} \delta_{j 3} \epsilon_{3 k l} e_{l}+\delta_{i 3} e_{j} \epsilon_{3 k l} e_{l}\right)-\frac{9}{4} e_{3}\left[(1-2 \hat{h}) R^{-3}\right. \\
& \left.-2 R^{-5}\right]\left(e_{j} \epsilon_{3 i k}+e_{i} \epsilon_{3 j k}\right)+\frac{9}{2} e_{3}\left[(1-\hat{h}) R^{-3}-R^{-5}\right] \delta_{i j} \epsilon_{3 k l} e_{l}-\frac{9}{2} e_{3}\left[5(1-\hat{h}) R^{-3}-7 R^{-5}\right] e_{i} e_{j}, \\
& \hat{M}_{E S, i j k l}^{\alpha \beta}=\frac{9}{5} R^{-7} \delta_{i 3} \delta_{j 3} \delta_{k l}-\frac{18}{5} e_{3}\left(5 R^{-5}-21 R^{-7}\right)\left(\delta_{i 3} \delta_{j 3} e_{k} \delta_{l 3}+\delta_{i 3} \delta_{j 3} \delta_{k 3} e_{l}\right)+\frac{9}{10} e_{3}\left(5 R^{-5}-14 R^{-7}\right)\left(e_{i} \delta_{j 3} \delta_{k l}+\delta_{i 3} e_{j} \delta_{k l}+\delta_{i l} \delta_{j 3} e_{k}\right. \\
& \left.+\delta_{i 3} \delta_{j l} e_{k}+\delta_{i 3} \delta_{j k} e_{l}+\delta_{i k} \delta_{j 3} e_{l}\right)+\frac{9}{5}\left[5 \hat{h}(1-\hat{h}) e_{3}^{2} R^{-3}+\left(1-10 e_{3}^{2}\right) R^{-5}-\left(2-21 e_{3}^{2}\right) R^{-7}\right]\left(\delta_{i k} \delta_{j 3} \delta_{l 3}+\delta_{i l} \delta_{j 3} \delta_{k 3}\right. \\
& \left.+\delta_{i 3} \delta_{j k} \delta_{l 3}+\delta_{i 3} \delta_{j l} \delta_{k 3}\right)-\frac{9}{10}\left[5 \hat{h}(1-\hat{h}) e_{3}^{2} R^{-3}+\left(1-5 e_{3}^{2}\right) R^{-5}-\left(1-7 e_{3}^{2}\right) R^{-7}\right]\left(\delta_{i l} \delta_{j k}+\delta_{i k} \delta_{j l}\right)+\frac{3}{20} \\
& \times\left[5\left(1-6 \hat{h}(1-\hat{h}) e_{3}^{2}\right) R^{-3}-6\left(1-5 e_{3}^{2}\right) R^{-5}+6\left(1-7 e_{3}^{2}\right) R^{-7}\right] \delta_{i j} \delta_{k l}-\frac{36}{5}\left[5 \hat{h}(1-\hat{h}) e_{3}^{2} R^{-3}+\left(1-15 e_{3}^{2}\right) R^{-5}\right. \\
& \left.-3\left(1-14 e_{3}^{2}\right) R^{-7}\right] \delta_{i 3} \delta_{j 3} \delta_{k 3} \delta_{l 3}-\frac{9}{5}\left[5\left(1-10 \hat{h}(1-\hat{h}) e_{3}^{2}\right) e_{3}^{2} R^{-3}-10\left(2-7 e_{3}^{2}\right) R^{-5}-\left(1-42 e_{3}^{2}\right.\right. \\
& \left.\left.+126 e_{3}^{4}\right) R^{-7}\right] \delta_{i j} \delta_{k 3} \delta_{l 3}-\frac{9}{5} e_{3}\left[5\left(1-20 \hat{h}(1-\hat{h}) e_{3}^{2}\right) R^{-3}-30\left(1-7 e_{3}^{2}\right) R^{-5}+84\left(1-6 e_{3}^{2}\right) R^{-7}\right]\left(e_{i} \delta_{j 3} \delta_{k 3} \delta_{l 3}\right. \\
& \left.+\delta_{i 3} e_{j} \delta_{k 3} \delta_{l 3}\right)+\frac{9}{20} e_{3}\left[5\left(1-20 \hat{h}(1-\hat{h}) e_{3}^{2}\right) R^{-3}-10\left(3-14 e_{3}^{2}\right) R^{-5}+14\left(2-9 e_{3}^{2}\right) R^{-7}\right] \times\left(e_{i} \delta_{j k} \delta_{l 3}+e_{i} \delta_{j l} \delta_{k 3}\right. \\
& \left.+\delta_{i k} e_{j} \delta_{l 3}+\delta_{i l} e_{j} \delta_{k 3}\right)-\frac{9}{20}\left[5\left(1-10 \hat{h}(1-\hat{h}) e_{3}^{2}\right) R^{-3}-10\left(1-7 e_{3}^{2}\right) R^{-5}+14\left(1-9 e_{3}^{2}\right) R^{-7}\right]\left(e_{i} e_{j} \delta_{k l}+\delta_{i j} e_{k} e_{l}\right) \\
& +\frac{9}{5}\left[25\left(1-14 \hat{h}(1-\hat{h}) e_{3}^{2}\right) e_{3}^{2} R^{-3}-70\left(2-9 e_{3}^{2}\right) R^{-5}-7\left(1-54 e_{3}^{2}+198 e_{3}^{4}\right) R^{-7}\right] e_{i} e_{j} \delta_{k 3} \delta_{l 3}+\frac{9}{10} e_{3} \\
& \times\left[5\left(1-10 \hat{h}(1-\hat{h}) e_{3}^{2}\right) R^{-3}-5\left(3-14 e_{3}^{2}\right) R^{-5}+14\left(2-9 e_{3}^{2}\right) R^{-7}\right]\left(\delta_{i j} e_{k} \delta_{l 3}+\delta_{i j} \delta_{k 3} e_{l}\right)-\frac{9}{40} \\
& \times\left[5\left(1-20 \hat{h}(1-\hat{h}) e_{3}^{2}\right) R^{-3}-20\left(1-14 e_{3}^{2}\right) R^{-5}+28\left(1-9 e_{3}^{2}\right) R^{-7}\right] \times\left(e_{i} \delta_{j l} e_{k}+\delta_{i l} e_{j} e_{k}+e_{i} \delta_{j k} e_{l}+\delta_{i k} e_{j} e_{l}\right) \\
& +\frac{9}{20}\left[5\left(1-20 \hat{h}(1-\hat{h}) e_{3}^{2}\right) R^{-3}-20\left(1-14 e_{3}^{2}\right) R^{-5}+28\left(2-27 e_{3}^{2}\right) R^{-7}\right] \times\left(e_{i} \delta_{j 3} e_{k} \delta_{l 3}+\delta_{i 3} e_{j} e_{k} \delta_{l 3}+e_{i} \delta_{j 3} \delta_{k 3} e_{l}\right. \\
& \left.+\delta_{i 3} e_{j} \delta_{k 3} e_{l}\right)-\frac{9}{10} e_{3}\left[25\left(1-14 \hat{h}(1-\hat{h}) e_{3}^{2}\right) R^{-3}-105\left(1-6 e_{3}^{2}\right) R^{-5}+126\left(2-11 e_{3}^{3}\right) R^{-7}\right]\left(e_{i} e_{j} e_{k} \delta_{l 3}+e_{i} e_{j} \delta_{k 3} e_{l}\right) \\
& -\frac{63}{5} R^{-7} \delta_{i 3} \delta_{j 3} e_{k} e_{l}-\frac{63}{10}\left(5 R^{-5}-9 R^{-7}\right)\left(\delta_{i 3} e_{j} e_{k} e_{l}+e_{i} \delta_{j 3} e_{k} e_{l}\right)+\frac{9}{20}\left[25\left(1-14 \hat{h}(1-\hat{h}) e_{3}^{2}\right) R^{-3}-70\left(1-9 e_{3}^{2}\right) R^{-5}\right. \\
& \left.+126\left(1-11 e_{3}^{2}\right) R^{-7}\right] e_{i} e_{j} e_{k} e_{l} \text {. }
\end{aligned}
$$

${ }^{1}$ J. Aizenberg, P. V. Braun, and P. Wiltzius, "Colloidal deposition controlled by electrstatic and capillary forces," Phys. Rev. Lett. 84, 2997 (2000).

${ }^{2}$ D. Leighton and A. Acrivos, "Measurement of shear-induced selfdiffusion in concentrated suspensions of spheres," J. Fluid Mech. 177, 109 (1987).

${ }^{3}$ R. Kubo, "The fluctuation-dissipation theorem," Rep. Prog. Phys. 29, 255 (1966).

${ }^{4}$ L. J. Durlofsky, J. F. Brady, and G. Bossis, "Dynamic simulation of hydrodynamically interacting particles," J. Fluid Mech. 180, 21 (1987).

${ }^{5}$ G. Bossis, A. Meunier, and J. D. Sherwood, "Stokesian dynamics simulations of particle trajectories near a plane," Phys. Fluids A 3, 1853 (1991). ${ }^{6}$ R. M. Jendrejack, D. C. Schwartz, J. J. de Pablo, and M. D. Graham, "Shear-induced migration in flowing polymer solutions," J. Chem. Phys. 120, 2513 (2004).

${ }^{7}$ B. Cichocki, R. B. Jones, R. Kutteh, and E. Wajnryb, "Friction and mobility for colloidal spheres in stokes flow near a boundary," J. Chem. Phys. 112, 2548 (2000).

${ }^{8}$ L. J. Durlofsky and J. F. Brady, "Dynamic simulation of bounded suspensions of hydrodynamically interaction particles," J. Fluid Mech. 200, 39 (1989).
${ }^{9}$ S. Kim and S. J. Karrila, Microhydrodynamics, 2nd ed. (Dover, New York, 2005).

${ }^{10}$ E. Lauga, W. R. DiLuzio, G. M. Whitesides, and H. A. Stone, "Swimming in circles: Motion of bacteria near solid boundaries," Biophys. J. 90, 400 (2006).

${ }^{11}$ J. Rotne and S. Prager, "Variational treatment of hydrodynamic interaction in polymers," J. Chem. Phys. 50, 4831 (1969).

${ }^{12} \mathrm{~J}$. R. Blake, "A note on the image system for a stokeslet in a no-slip boundary," Proc. Cambridge Philos. Soc. 70, 303 (1971).

${ }^{13}$ J. R. Blake and A. T. Chwang, "Fundamental singularities of viscous flow," J. Eng. Math. 8, 23 (1974).

${ }^{14}$ E. Lauga and T. M. Squires, "Brownian motion near a partial-slip boundary," Phys. Fluids 17, 103102 (2005).

${ }^{15}$ J. J. L. Higdon, "The generation of feeding currents by flagellar motions," J. Fluid Mech. 94, 305 (1979).

${ }^{16}$ T. N. Phung, J. F. Brady, and G. Bossis, "Stokesian dynamics simulations of Brownian suspensions," J. Fluid Mech. 313, 181 (1996).

${ }^{17}$ S. H. Lee, R. S. Chadwick, and L. G. Leal, "Motion of a sphere in the presence of a plane interface. Part 1 . An approximate solution by generalization of the method of Lorentz," J. Fluid Mech. 93, 705 (1979).

${ }^{18}$ E. R. Dufrense, D. Altman, and D. G. Grier, "Brownian dynamics of a sphere between parallel walls," Europhys. Lett. 53, 264 (2001). 